\title{
Interpersonal Sources of Conflict in Young People with and without Mild to Moderate Intellectual Disabilities at Transition from Adolescence to Adulthood
}

P. Larkin, A. Jahoda, K. MacMahon and C. Pert

Psychological Medicine, Gartnavel Royal Hospital, University of Glasgow, Glasgow, UK

Accepted for publication 6 May 2011

Background Interpersonal conflict is a source of stress and contributes to poor mental health in people with mild to moderate intellectual disabilities. Understanding the contexts in which conflict typically occurs can better equip services to help people with such difficulties. However, existing studies into the contexts of conflict have included participants with wide-ranging ages and may not reflect the experiences of young adults in particular.

Materials and Methods Twenty-six young adults (16-20 years) with intellectual disabilities and 20 non-disabled young adults completed a semi-structured interview about a recent experience of interpersonal conflict. Participants were asked to describe their beliefs and feelings about the event and their subsequent response. Results Participants with intellectual disabilities were more likely to encounter conflict with strangers or peers outside their friendship group and to describe incidents of aggression than non-disabled participants. They were also more likely to characterize the other person globally as 'bad' and to perceive the other's actions as being personally directed at them. Young women with intellectual disabilities were less likely to describe responding aggressively to incidents.

Conclusions Findings suggest that young adults with intellectual disabilities are often the target of overt aggression from those outside their inner social sphere, while their non-disabled peers are more likely to experience conflict with people close to them. Young adults with intellectual disabilities may also be more likely to feel victimized by interpersonal conflict. Implications of these findings and limitations of the study are discussed.

Keywords: intellectual disabilities, interpersonal conflict, transition to adulthood

\section{Introduction}

\section{Background}

Dealing with social conflict can be a particularly difficult experience for many people with mild to moderate intellectual disabilities. Studies have found that adults in this group identify difficult social interactions as occurring more often and as being more stressful than many other negative life events (Bramston et al. 1999; Hartley \& Maclean 2009). In fact, negative social interactions such as disrespectful treatment and victimisation appear to be more common in the everyday lives of people with intellectual disabilities than in the non-disabled population (Sobsey 1994; Jahoda \& Markova 2004; Levy \& Packman 2004). In addition to causing psychological distress, exposure to such social stressors has been linked to health and mental health problems in this group including depressive symptoms and somatic complaints (Lunsky \& Benson 2001; Emerson 2010).

One of the most important reasons for investigating the nature of interpersonal conflict in people with intellectual disabilities is that a significant minority of this population has problems with aggression. Such problems can have profoundly detrimental effects on the lives of the individuals displaying the aggressive behaviour and on the lives of those around them (Murphy 1993; Bruininks et al. 1994; Taylor et al. 2002; Tyrer et al. 
2006). However, little is known about the everyday social interactions that typically provoke aggression in people with intellectual disabilities.

\section{Social conflict experienced by people with intellectual disabilities}

To date, three studies have examined the interpersonal sources of conflict in adults with intellectual disabilities. Benson \& Fuchs (1999) interviewed a group of frequently aggressive adults from Illinois, USA, about recent social interactions at home and at work that had angered them. They found that conflict at work was typically with coworkers and involved aggression. At home, conflicts were most commonly with peers and siblings. More recently, Hunter et al. (2010) used data collected from anger-management interventions in the UK to identify the most common social experiences for 10 adults that led to significant feelings of anger. They found that the most widely reported incidents of this type were 'minor annoyances' such as 'nagging' or being 'told off'. However, the incidents rated as being most aggravating were of personal abuse, including being (i) shouted at, (ii) physically threatened and (iii) 'picked on'. Such incidents were also relatively widespread, with six of the 10 participants reporting being called names, five participants stating they had been shouted at and four participants reporting being physically threatened.

A limitation of both Hunter et al.'s (2010) and Benson \& Fuchs (1999) studies is that they included neither non-aggressive comparison groups nor individuals without intellectual disabilities. Consequently, it is not clear whether the patterns observed in these individuals with problems of aggression are different from their non-aggressive peers or from individuals without intellectual disabilities of a similar background. With this in mind, MacMahon et al. (2006b) re-analysed interview data with 53 aggressive and non-aggressive adults with intellectual disabilities that had been collected by Jahoda et al. (1998). In the latter study, participants were asked to describe a recent situation of conflict that continued to elicit negative emotions on recall. Re-analysis by MacMahon et al. (2006b) indicated that the most common source of conflict in both groups was interaction with fellow service users. Additionally, the authors re-analysed the participants' interpersonal perceptions of the person with whom they were in conflict. They found that both groups tended to believe they were being belittled or treated in a condescending manner.

\section{Young adults with intellectual disabilities}

All three studies described above found that incidents of conflict were often with peers and often involved aggression. However, the experiences of adolescents and younger adults that spend the majority of their daytime at school or college, rather than adult resource centres or work settings, are not well represented in these studies. This is of significance as transition to adulthood may be a particularly difficult period for people with intellectual disabilities. Young people with intellectual disabilities in full-time education may be particularly likely to encounter bullying, which, amongst other things, is associated with poor mental health (Nabuzoka \& Smith 1993; Lunsky \& Benson 2001). Also, at a developmental stage typified by increased independence in most groups, young adults with intellectual disabilities may become more aware of, and concerned about, relative limitations to their own independence. Such limitations may either result from specific cognitive limitations or be the result of intervention by others (most typically parents) who may be concerned for the well-being of the individual.

Theoretically, these factors (of awareness of limited independence in comparison with non-disabled peers) could result in some individuals perceiving themselves in a more negative light, or, indeed, to the perception of others as behaving in a manner that does not give them sufficient credit as an individual who is moving from adolescence to adulthood. Subsequently, an enervated sense of self could, in turn, lead some to depression, frustration, or even aggressive behaviour (Jahoda et al. 2001; Cheng \& Furnham 2003). Problems stemming from a vulnerable sense of self or from low self-esteem could be further compounded by frequent experiences of victimization. Given these concerns, the present study focuses on exploring interpersonal conflict in individuals in the transition between adolescence and adulthood (16-20 years).

\section{Aims}

The study aimed to identify key contextual features of conflict for young people with intellectual disabilities. It also examined participants' perceptions of these experiences, as well as reports of their subsequent emotions and behavioural responses. Accounts were obtained through semi-structured interviews.

A semi-structured interview methodology offered several specific advantages. Such methods can generate a rich data set suited to exploratory research with broadly defined research aims. Crucially, in using an 
interview that addresses actual experiences of angerprovoking events, it was possible to evoke emotional states similar to those felt during such experiences, thereby increasing the ecological validity of the data gathered. Finally, the chosen measure, the CognitiveEmotive Behavioural Assessment (CEBA), has been proven to be engaging and accessible for people with intellectual disabilities, thus increasing the probability that data gathered will accurately reflect the experiences of participants.

To examine the extent to which common features of conflict for young people with intellectual disabilities are specific to this group, a comparison group of typically developing young people was included. Also, research suggests possible gender differences in the conflict experiences and preferred responses of adolescents (Lindeman et al. 1997; Rudolph \& Hammen 1999). Specifically, there is reason to anticipate men to report more aggressive responses to conflict than women (Archer 2004). For this reason, the present study included additional analyses to examine whether gender differences are present in young adults with intellectual disabilities.

Previous research suggested that victimization, the involvement of others with intellectual disabilities (in situations of conflict), stigma and aggression from others were likely to emerge as key themes from this study. On the basis of the limited existing research, the following research questions were asked concerning reports of conflict by young people with and without intellectual disabilities:

1. Do the contextual features of conflict differ between the two groups, in terms of (i) the type of event, (ii) who the conflict is with, and (iii) the location?

2. Do the interpersonal perceptions of two groups differ, in terms of (i) their attribution of intent and (ii) their appraisal of the other?

3. Do the two groups report behaving differently?
Further hypotheses were offered concerning frequently aggressive and non-aggressive participants:

1. Aggressive participants will be more likely to attribute hostile intent to those they are in conflict with.

2. Aggressive participants will be more likely to report responding aggressively.

Finally, in line with past research on gender differences, it was hypothesized that male participants will report more aggressive responses than female participants.

\section{Methods}

Ethical approval for the study was granted by the Greater Glasgow and Clyde NHS Ethics Committee.

\section{Participants}

Twenty-six participants with mild to moderate intellectual disabilities were recruited from two further education colleges that provide specialist courses for young people with additional learning needs. The non-disabled group comprised 20 participants from two colleges, one youth club and one secondary school in central Scotland.

Recruitment commenced with brief presentations to selected groups and classes of young people, explaining the aims of the study and what participation would entail. Those interested in participating in the study then returned a reply slip to a designated member of staff. Unfortunately, researchers do not have a record of attendance for each presentation and, consequently, it is not possible to say what proportion of those present at the presentations consented to participate in the study.

The participants' socio-demographic details are displayed in Table 1. All participants lived with their parents, were aged between 16 and 20 years of age and were planning to leave full-time education by the end of

Table I Participant details

\begin{tabular}{|c|c|c|c|c|c|c|}
\hline Group & $N$ & $\begin{array}{l}\text { Mean } \\
\text { age }\end{array}$ & $\begin{array}{l}\text { Mean Carstairs } \\
\text { deprivation score }\end{array}$ & $\begin{array}{l}\text { Mean IQ } \\
\text { estimate (WASI) }\end{array}$ & Gender & $\begin{array}{l}\text { Frequently } \\
\text { aggressive }\end{array}$ \\
\hline ID & 26 & $18.6(\mathrm{SD}=0.95)$ & $3.8(\mathrm{SD}=5.65)$ & $62.12(\mathrm{SD}=8.01)$ & $\mathrm{M}=15 ; \mathrm{F}=11$ & $\mathrm{Ag}=12 ; \mathrm{Nag}=13$ \\
\hline ND & 20 & $\begin{array}{l}17.1(\mathrm{SD}=0.97) \\
P \leq 0.01 \\
\text { Mann-Whitney } \\
U=73.0\end{array}$ & $\begin{array}{l}1.9(\mathrm{SD}=4.59) \\
P=0.263 \\
\text { Mann-Whitney } \\
U=209.5\end{array}$ & $\begin{array}{l}93.6(\mathrm{SD}=10.53) \\
P \leq 0.01 \\
\text { Mann-Whitney } \\
U \leq 0.01\end{array}$ & $\begin{array}{l}\mathrm{M}=11 ; \mathrm{F}=9 \\
\chi_{1}^{2}=0.33, P=0.855\end{array}$ & $\begin{array}{l}\mathrm{Ag}=2 ; \mathrm{Nag}=18 \\
\chi_{1}^{2}=6.98 P=0.008\end{array}$ \\
\hline
\end{tabular}

ID, intellectual disability; ND, non-disabled; WASI, Wechsler Abbreviated Scale of Intelligence. 
the following academic year. Additional Supports Needs staff at colleges identified individuals receiving support from their department. These staff also verified that potential participants had sufficient receptive and expressive language skills to engage in the interview. Researchers considered that some individuals with autism spectrum disorders might struggle to engage with interview items regarding interpersonal perception. Therefore, staff were asked to indicate whether potential participants had been diagnosed with autism spectrum disorders, and those individuals were excluded from the study. Seven potential non-disabled participants were also excluded on the basis of Wechsler Abbreviated Scale of Intelligence (WASI) scores below the threshold for borderline intellectual disability.

\section{Measures and interview}

\section{Semi-structured interview}

The semi-structured interview was adapted from the CEBA interview previously used in studies with individuals with intellectual disabilities (Trower et al. 1988; Jahoda et al. 1998). The interview questions followed an 'ABC' format where participants were asked to describe (A) an 'activating event' or incident of interpersonal conflict, (B) their beliefs about what was happening, which lead to $(C)$ the consequent emotions and behaviour. The method involved asking the participants to recall a recent incident of interpersonal conflict which still aroused feelings of anger or other negative emotions. Next, participants were asked to describe the incident in their own words including where it took place, who was involved and their view of what happened (A). They were then asked to describe their emotions at the time and their subsequent responses (C). Finally, the interviewer tried to get the participant to hold this emotion in mind when covering the following topics: (i) their perceptions of the intent of the other person and (ii) how they viewed the other person (B). In addition to the original CEBA items, participants were asked to describe any techniques employed to control their emotions during or immediately after the incident. Where participants offered more than one answer to questions, they were asked to choose the answer that was most important to them.

\section{Wechsler Abbreviated Scale of Intelligence}

The WASI provides an estimate of general intellectual ability by testing the participants' vocabulary and matrix reasoning skills. The WASI is an abbreviated version of the Wechsler Adult Intelligence Scale-III (WAIS-III; Psychological Corporation, 1999). Correlation scores between the WASI and WAIS-III are acceptable at 0.87 for vocabulary, 0.66 for matrix reasoning and 0.87 overall.

\section{Checklist of Challenging Behaviour (CCB)}

Staff members who had known the participants for at least 6 months completed the 'Aggression' section of the CCB with a member of the research team (Harris 1993). The CCB was used to assign participants to aggressive and non-aggressive subgroups in order to conduct post hoc comparisons. Participants reported to have enacted five serious acts of verbal or physical aggression over the preceding 6 months were classified as being frequently aggressive. The CCB was developed specifically for use with people with intellectual disabilities.

\section{Procedure}

All participant interviews took place in private rooms at the recruitment site. Participants were assured that the researchers were interested in their feelings and opinions about conflict and that there were no right or wrong answers. Permission was requested from participants to allow the interviews to be recorded using digital media. Permission was also sought to interview staff at a later date about their recent behaviour.

Each interview began with an informal conversation to build a rapport between the researcher and participant and to put the participant at ease. Participants then completed the interview with the researcher as outlined above. Finally, participants completed the two-subtest form of the WASI. The WASI was completed after the main interview because it was judged to be contradictory to the open spirit of the interview that sought to explore the experiences of participants.

Once the data had been collected from the participants, their permission was sought to speak with a staff member. These staff members were asked to complete the aggression section of the CCB with a member of the research team (Harris 1993). As all staff members who completed the CCB had known the participants for at least 6 months, they were all able to report confidently on the participants' pattern of behaviour.

\section{Analysis strategy}

The participants'responses to each of the interview questions were transcribed verbatim. Three participants did 
not consent to interviews being recorded on a digital voice recorder. In these instances, responses were recorded onto a pre-prepared response sheet by the researcher during the interview.

Content analysis was considered to be more appropriate than other qualitative methods of analysis, such as interpretative phenomenological analysis, because the data consisted of participants' responses to specific interview questions rather than a more open dialogue about particular topics.

Once transcripts had been content analysed, answers to each interview item were grouped into categories that reflected the different responses given by participants. To elucidate how this was carried out, it may be useful to consider an example of how these categories were developed for responses to one of the interview questions. In one case, when asked how he reacted to being punched by his brother, a participant replied ' $\mathrm{I}$ 'd hit him back'. In response to the same question, another participant, who reported being unfairly told off by her mother, claimed 'I just kept my mouth shut'. The first response, where the boy hit his brother, was provisionally coded as 'aggressive', while the second response was coded as 'passive'. After assigning provisional categories to the remainder of participants' responses, it became apparent that a meaningful coding frame for responses to this question would be the three categories 'aggressive', 'passive' and 'assertive'. Responses that had been provisionally coded as other categories were then recoded as one of these three chosen categories.

A second independent rater was asked to use the coding frames to categorize the responses of $26 \%$ of the sample. The subsample was chosen at random and included seven participants with intellectual disabilities and five non-disabled participants. Overall agreement between the two raters was 93\% across all interview items. Inter-rater reliability analyses were conducted for responses to each of the six interview items (' $a$ ' to ' $f$ ') using kappa statistics. These analyses yielded the following results: (a) $\kappa=1,(P=0.001)$; (b) $\kappa=0.824,(P=0.004)$; (c) $\kappa=1,(P=<0.001) ;(\mathrm{d}) \kappa=0.698,(P=0.003) ;(\mathrm{e}) \kappa=0.860$, $(P \leq 0.001)$; and (f) $\kappa=0.845,(P \leq 0.001)$.

Two-tailed chi-square comparisons of coded responses to each question were carried out between groups. Aggression scores obtained from teachers or lecturers were used to assign participants with intellectual disabilities to aggressive and non-aggressive subgroups. Comparisons were made between these subgroups. Finally, the responses of male and female members of each group were also compared.

\section{Results}

Comparisons between the responses of the two groups are presented first, followed by comparisons between men and women and between frequently aggressive and non-aggressive individuals.

\section{Comparisons between individuals with and without intellectual disabilities}

\section{A. Activating event}

Type of event. The results summarized in Table 2a show that participants with intellectual disabilities cited significantly more incidents of verbal or physical aggression than the non-disabled group. Over half of participants with intellectual disabilities described situations of verbal or physical aggression, while only one of the 20 non-disabled participants described such situations.

The other person involved. Table $2 \mathrm{~b}$ shows that a significant difference was also found between the two groups in terms of the people with whom participants were in conflict. The large majority of non-disabled participants reported conflict with people they were close to. Conversely, most people with intellectual disabilities reported being in conflict with people they were less close to such as strangers, neighbours or peers outwith their friendship group.

Location of event. Table $2 \mathrm{c}$ indicates that there were no significant differences between the two groups in terms of where conflict took place. However, it is worth noting that half of the participants with intellectual disabilities encountered conflict at school/college/work compared with four of the non-disabled participants. Several participants in each group described incidents that took place over the phone (four of 26 participants with intellectual disabilities, five of 20 non-disabled participants) or via computer (one participant with and one participant without intellectual disabilities) rather in actual locations. The use of telephones and modern media may be an interesting feature of conflict in young people with and without intellectual disabilities. However, it was decided that these responses were categorically different from those designating the actual geographical location of experiences of conflict and would thus be excluded from the present analyses. 


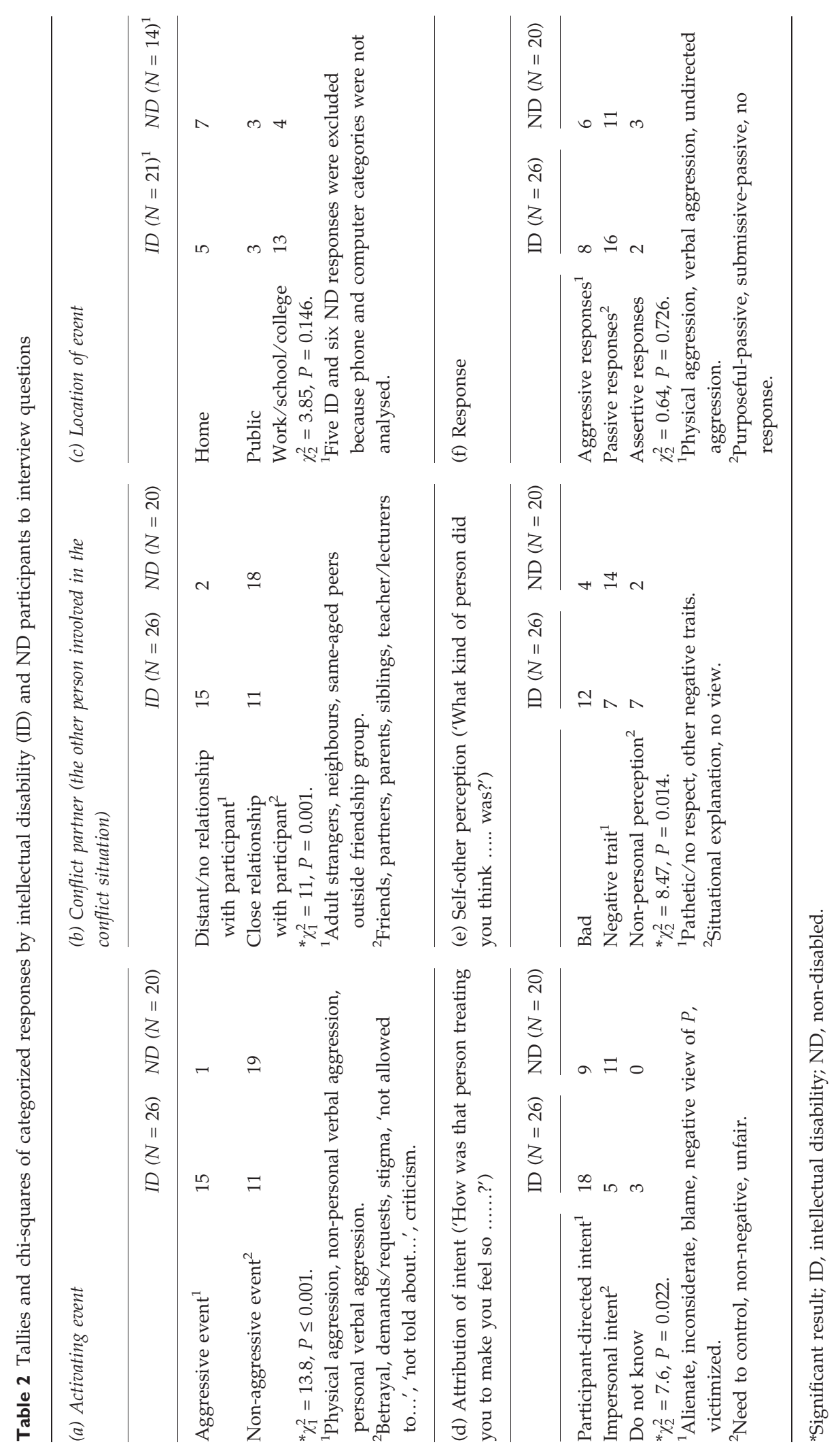

(C) 2011 Blackwell Publishing Ltd, 25, 29-38 


\section{B. Interpersonal beliefs}

Attribution of intent. Table $2 \mathrm{~d}$ shows that there was a significant group difference in the type of motive that participants attributed to their conflict partners. While most of the participants with intellectual disabilities viewed their conflict partners' actions as being directed at them personally (alienate, inconsiderate, blame, negative view and victimized), over half of the non-disabled group attributed motives unrelated to the participant (conflict partner's need to control the situation, no negative motive and unfair situation).

Self-other beliefs (appraisal of other). Fourteen of the 20 non-disabled participants attributed the other's behaviour to a specific negative trait on the other's part. However, only seven of the 26 participants with intellectual disabilities responded similarly with 12 instead appraising the other globally as a 'bad' person. Comparisons between the three categories shown in Table $2 \mathrm{e}$ reveal these differences to be significant.

\section{Response}

As described in Table $2 \mathrm{f}$, no statistically significant group differences emerged, with both groups reporting equivalent numbers of passive, assertive and aggressive responses. Of the 46 participants in both groups, only five responded assertively.

\section{Aggressive and non-aggressive subgroups}

Where staff reported five or more significant incidents of verbal or physical aggression over the preceding 6 months, participants were categorized as frequently aggressive. As only two non-disabled participants were considered to be aggressive, it was not possible to conduct comparisons between aggressive and nonaggressive participants. However, 12 of the 26 participants with intellectual disabilities met the criteria for frequent aggression, and comparisons were made between aggressive and non-aggressive participants. No significant differences were found between aggressive and non-aggressive subgroups.

\section{Gender}

Both groups had sufficient numbers of men and women to conduct gender comparisons within groups (ID $=15$ men, 11 women; $\mathrm{ND}=11$ men, 9 women). A statistically significant difference in response category emerged between male and female participants with intellectual disabilities $\left(\chi_{2}^{2}=7.052, P=0.029\right)$. Seven of the 15 male participants responded aggressively to the event compared with only one of the eleven female participants. The remaining 10 women responded passively, while only six of the 15 men responded passively and two responded assertively. Gender comparisons of nondisabled participants revealed no significant group differences.

\section{Discussion}

The results suggest that there might be differences between the typical interpersonal conflict experiences of young people with and without intellectual disabilities. Participants with intellectual disabilities frequently reported aggressive incidents, which is broadly in line with findings from samples with wider age ranges (Benson \& Fuchs 1999; MacMahon et al. 2006a,b). Perhaps a more novel finding is that the participants with intellectual disabilities mainly reported conflict with strangers and peers outwith their friendship group. They were also far likelier to cite conflict with strangers than their non-disabled peers were. As people with intellectual disabilities often have relatively restricted social lives, it might be that a greater proportion of their social interactions, including incidents of conflict, are with people outside their social network. (Myers et al. 1998; Ager et al. 2001).

The study also found that young people with intellectual disabilities made significantly different attributions about the experiences they discussed. For one, they were more likely to feel like the person with whom they were in conflict was targeting them personally. It is quite plausible that participants in this group were indeed being singled-out more often than people in the non-disabled group. However, these results may also suggest that young people with intellectual disabilities are particularly vulnerable to feeling threatened or aggravated by difficult social situations. Many people with intellectual disabilities have experiences of stigma or social exclusion, either of which can have a negative impact on how they view themselves (Szivos-Bach 1993; Foundation for People with Learning Disabilities 2001; Cooney et al. 2006). Furthermore, experiences of subordination may increase emotional reactivity to stressful situations, including interpersonal conflict (Boyce 2004). It may be that many young people with intellectual disabilities develop emotional and cognitive tendencies that make it harder to defuse difficult social situations and 
thus put them at increased risk of being drawn into conflict. Perhaps more worryingly, previous studies have found that both the propensity to perceive hostility in others and emotional arousal could be key factors underpinning problems of aggression (Pert et al. 1999; Jahoda et al. 2006; MacMahon et al. 2006a,b). It is possible that this could partially explain the relatively large proportion of frequently aggressive individuals in the group with intellectual disabilities. It could also shed some light on why frequent aggression is a problem for a significant minority of people with intellectual disabilities (Tyrer et al. 2006).

Participants with intellectual disabilities were more likely to make negative, generalizing attributions about their conflict partners. Regarding someone as a 'bad person' could be seen as taking a less empathetic view of that individual than, for example, identifying specific shortcomings in their character or behaviour. Feeling less empathy for their conflict partners could make it even more difficult for some people in this group to prevent conflicts from escalating to more serious confrontations. Interestingly, one might expect people to feel less empathy for individuals outside their circle of trust. This may be an alternative explanation for why most of the participants with intellectual disabilities reported incidents with people less close to them.

There were no differences found in the number of aggressive responses to conflict given by the two groups. This is perhaps surprising given that a far greater proportion of the group with intellectual disabilities were found to have problems of aggression. Similarly, this seems at odds with findings discussed above where participants with intellectual disabilities described more incidents of aggression and appeared to have more negative cognitions about their experiences. It is possible that some participants, conscious of their aggressiveness, felt that this question put their behaviour under scrutiny. Consequently, some may have felt reluctant to give aggressive responses and, instead, may have described more socially desirable responses.

It was surprising that the aggressive group with intellectual disabilities did not offer more aggressive responses than the non-aggressive group. Moreover, previous findings that such aggressive individuals attribute hostile intent more readily than other individuals were not replicated (Pert et al. 1999; Basquill et al. 2004; Jahoda et al. 2006). However, given the small number of participants in the present study, these findings should be interpreted with a degree of caution.

An additional finding was that male participants with intellectual disabilities were more likely to respond aggressively than the women in the group. This is commensurate with previous findings that adolescent males are more overtly aggressive than adolescent females (Archer 2004). It is noteworthy that this effect was not observed in the non-disabled group. However, this may simply reflect the relatively small size of the non-disabled group.

\section{Limitations of the study}

A larger sample may have clarified non-significant trends and provided a broader range of responses to the interview questions. There were also a number of socio-demographic differences between the groups that could offer alternative explanations for some of the findings. The participants with intellectual disabilities were from more deprived backgrounds than the non-disabled group. Also, they were on average one and a half years older and included more frequently aggressive individuals. While all participants with intellectual disabilities were college goers, the non-disabled group included nine school pupils and one youth club attendee.

Although the interviewer was blind to aggressiveness, it was not possible for the interviewer to be blind to intellectual disability. However, any interviewer bias was minimized by ensuring that the initial questions of all interview items were phrased consistently over interviews.

Finally, the data presented in the present study is based on a basic analysis of the interview data. In future research, it may be interesting to carry out a more in-depth qualitative analysis of interpersonal conflict given by participants.

\section{Future research}

Research into the psychosocial sources of aggression in people with intellectual disabilities frequently utilizes vignettes depicting scenes of social conflict. The storylines for these vignettes are often developed without any evidence base. There is therefore a risk that the chosen scenarios might either include irrelevant features or exclude highly salient features of conflict experienced by a given group. The present findings may facilitate the development of vignettes for future research that are relevant to the everyday experiences of young adults with intellectual disabilities. With further development, such vignettes could also serve as a useful tool in assessing reactive aggressiveness in young adults with intellectual disabilities. 


\section{Conclusion}

Young people with intellectual disabilities may be more likely to feel victimized by experiences of social conflict. It appears that many young people with intellectual disabilities develop cognitive and emotional tendencies that may exacerbate situations of conflict or even lead to aggression. Such tendencies could be rooted in certain life experiences common to this group such as stigma or social isolation. Future research may seek to explore how, and when, such tendencies develop over the lifespan by continuing to examine experiences and perceptions of conflict at specific developmental stages. While certain psychological tendencies may well escalate situations of conflict, it also seems that young adults with intellectual disabilities are more likely to be targeted by others for verbal and physical abuse. Therefore, future studies may also seek to identify factors that facilitate resilience in the face of more serious confrontation.

\section{Correspondence}

Any correspondence should be directed to Peter Larkin, Psychological Medicine, Gartnavel Royal Hospital, University of Glasgow, Glasgow G12 0XH, UK (e-mail: p.larkin@clinmed.gla.ac.uk).

\section{References}

Ager A., Myers F., Kerr P., Myles S. \& Green A. (2001) Moving home: social integration for adults with learning disabilities resettling into community provision. Journal of Applied Research in Learning Disabilities 14, 392-400.

Archer J. (2004) Sex differences in aggression in real-world settings: a meta-analytic review. Review of General Psychology 8, 291-322.

Basquill M. F., Maguth Nezu C., Nezu A. M., Klein T. L. \& MacLean . W. E. Jr (2004) Aggression-related hostility bias and social problem-solving deficits in adult males with mental retardation. American Journal on Mental Retardation 109, 255-263.

Benson B. A. \& Fuchs C. (1999) Anger arousing situations and coping responses of aggressive adults with intellectual disability (n1). Journal of Intellectual and Developmental Disability 24, 207-215.

Boyce W. T. (2004) Social stratification, health, and violence in the very young. In: Youth Violence: Scientific Approaches to Prevention (eds J. Devin, J. Gilligan, K. A. Miczek, R. Shaikh \& D. Pfaff), pp. 47-68. New York Academy of Sciences, New York, NY, USA.

Bramston P., Fogarty G. \& Cummins R. A. (1999) The nature of stressors reported by people with an intellectual disability. Journal of Applied Research in Intellectual Disabilities 12, 1-10.
Bruininks R.H., Olson K.M., Larson S.A. \& Lakin K.C. (1994) Challenging behaviors among persons with mental retardation in residential settings. In: Destructive Behavior in Developmental Disabilities: Diagnosis and Treatment (eds T. Thompson \& D.B. Gray), pp. 24-48. viii, 327. Sage Publications, Inc., Thousand Oaks, CA.

Cheng H. \& Furnham A. (2003) Attributional style and self-esteem as predictors of psychological well being. Counselling Psychology Quarterly 16, 121-130.

Cooney G., Jahoda A., Gumley A. \& Knott F. (2006) Young people with intellectual disabilities attending mainstream and segregated schooling: perceived stigma, social comparison and future aspirations. Journal of Intellectual Disability Research 50, 432-444.

Emerson E. (2010) Self-reported exposure to disablism is associated with poorer self-reported health and well-being among adults with intellectual disabilities in England: a cross-sectional survey. Public Health 124, 682-689.

Foundation for People with Learning Disabilities (2001) Learning Disabilities: The Fundamental Facts. FPLD, London.

Harris P. (1993) The nature and extent of aggressive behavior amongst people with learning difficulties (mental handicap) in a single health district. Journal of Intellectual Disability Research 37, 221-242.

Hartley S. L. \& Maclean W. E. (2009) Stressful social interactions experienced by adults with mild intellectual disability. American Journal on Intellectual and Developmental Disabilities 114, 71-84.

Hunter C., Nadany S. \& Cragg R. (2010) The Birmingham provocation hierarchy: for adults with mild to borderline learning disabilities. Clinical Psychology Forum 207, 25-29.

Jahoda A. \& Markova I. (2004) Coping with social stigma. People with intellectual disabilities moving from institutions and family home. Journal of Intellectual Disability Research 48, 719-729.

Jahoda A., Pert C., Squire J. \& Trower P. (1998) Facing stress and conflict: a comparison of the predicted responses and self-concepts of aggressive and non-aggressive people with intellectual disability. Journal of Intellectual Disability Research 42, 360-369.

Jahoda A., Trower P., Pert C. \& Finn D. (2001) Contingent reinforcement or defending the self? A review of evolving models of aggression in people with mild learning disabilities. British Journal of Medical Psychology 74, 305-321.

Jahoda P., Pert C. \& Trower P. (2006) Frequent aggression and attribution of hostile intent in people with mild to moderate mental retardation: an empirical investigation. American Journal on Mental Retardation 111, 90-99.

Levy H. \& Packman W. (2004) Sexual abuse prevention for individuals with mental retardation: considerations for genetic counselors. Journal of Genetic Counseling 13, 189-205.

Lindeman M., Harakka T. \& Keltikangas-Jarvinen L. (1997) Age and gender differences in adolescents' reactions to conflict situations: aggression, prosociality, and withdrawal. Journal of Youth and Adolescence 26, 339-351. 
Lunsky Y. \& Benson B. A. (2001) Association between perceived social support and strain, and positive and negative outcomes for adults with mild intellectual disability. Journal of Intellectual Disability Research 45, 106-114.

MacMahon K. M. A., Jahoda A., Espie C. A, Broomfield N. A \& Niall M. (2006a) The influence of anger-arousal level on attribution of hostile intent and problem solving capability in an individual with a mild intellectual disability and a history of difficulties with aggression. Journal of Applied Research in Intellectual Disabilities 19, 99-107.

MacMahon K., Jahoda A. \& Pert C. (2006b) Sources of angerarousal for individuals with mild to moderate intellectual disabilities with and without difficulties with frequent aggression. Abstract from Seattle Club Conference, Kendall.

Murphy G. (1993) The treatment of challenging behaviour in people with learning difficulties. In: Violence: Basic and Clinical Science (eds C. Thompson \& P. Cowan), pp. 171-185. Butterworth-Heineman, Oxford.

Myers F., Ager A., Kerr P. \& Myles S. (1998) Outside looking in? Studies of the community integration of people with learning disabilities. Disability and Society 13, 389-413.

Nabuzoka D. \& Smith D. K. (1993) Sociometric status and social behaviour of children with and without learning difficulties. Journal of Child Psychology and Psychiatry 34, 14351448.

Pert C., Jahoda A. \& Squire J. (1999) Attribution of intent and role-taking: cognitive factors as mediators of aggression with people who have mental retardation. American Journal on Mental Retardation 104, 399-409.

Psychological Corporation (1999) Wechsler Abbreviated Scale of Intelligence (WASI). Psychological Corporation, San Antonio, TX.

Rudolph K. D. \& Hammen C. (1999) Age and gender as determinants of stress exposure, generation, and reactions in youngsters: a transactional perspective. Child Development 70, 660-677.

Sobsey R. (1994) Violence and Abuse in the Lives of People with Disabilities: The End of Silent Acceptance? xxii, 444 pp. Paul H Brookes Publishing, Baltimore, MD.

Szivos-Bach S.E. (1993) Social comparisons, stigma and mainstreaming: the self esteem of young adults with a mild mental handicap. Mental Handicap Research 6, 217-236.

Taylor J., Novaco R., Gillmere B. \& Thorne I. (2002) Cognitivebehavioural treatment of anger intensity among offenders with mental retardation. Journal of Applied Research in Mental Retardation 15, 151-165.

Trower P., Casey A. \& Dryden W. (1988) Cognitive-Behavioural Counselling in Action. viii, 152 pp. Sage Publications, Inc., Thousand Oaks, CA.

Tyrer F., McGrother C. W., Thorp C. F., Donaldson M., Bhaumik S., Watson J. M. \& Hollin C. (2006) Physical aggression towards others in adults with learning disabilities: prevalence and associated factors. Journal of Intellectual Disability Research 50, 295-304. 